\title{
AUSENCIAS MIOCLÔNICAS: ESTUDO COMPARATIVO DA POTENCIA ANTICONVULSIVANTE DO NITRAZEPAM (MOGADON) E DO DIAZEPAM (VALIUM)
}

\author{
Michel PierRe Lison
}

A ação do diazepam sôbre ausências típicas ${ }^{3,4,13}$ é de interêsse embora outras drogas também ajam satisfatòriamente sôbre esta forma eletro-clinica de epilepsia. Gastaut e col. 3,4 assinalaram a ação do diazepam sôbre outras formas de epilepsia generalizada, incluindo as ausências mioclônicas para as quais as dionas e succinimidas são pouco ativas ou ineficazes.

A ação do nitrazepam sôbre a epilepsia generalizada não convulsiva ainda não é perfeitamente conhecida. Observações colhidas na literatura $6,9,12$ e observações pessoais não publicadas indicam que o nitrazepam é ativo nas ausências típicas, mas talvez não tanto quanto as succinimidas e a trimetadiona. As referências sôbre o efeito do nitrazepam nas ausências mioclônicas são poucas e as casuísticas insuficientes para conclusões definitivas. A ação bastante acentuada do nitrazepam sôbre as epilepsias mioclônicas do lactente e da criança ${ }^{5,8,9,11,14,15}$ e sôbre certas formas de ausências atípicas (Biolley ${ }^{2}$; observações pessoais) nos levou a investigar seu efeito nas ausências mioclônicas.

Markham ${ }^{9}$ assinala que o efeito do nitrazepam está, em grande parte, ligado à presença atual ou remota de espasmos infantis. Crises de diversas categorias, desde que associadas aos espasmos infantis, seriam beneficiadas. Adultos ou crianças com diversos tipos de manifestações epiléticas, generalizadas ou focais, sem antecedentes de mioclonias maciças, não apresentariam melhoras significativas.

Justificam-se, pois, estudos comparativos sôbre a ação anticonvulsivante dos derivados benzodiazepínicos na epilepsia generalizada da criança, do adolescente e co adulto jovem. A avaliação correta dos efeitos anticonvulsivantes próprios de cada droga permitirá estabelecer, com maior rigor, bases racionais para a terapêutica adequada das diversas formas de epilepsia.

Estudos semelhantes continuam sendo executados com drogas clássicamente utilizadas ${ }^{1,16}$.

Nota do autor - Este trabalho constitui parte da tese apresentada para concurso à Docência Livre de Clínica Neurológica na Faculdade de Medicina de Ribeirão Prêto da Universidade de São Paulo. 


\section{CASUfSTICA E MÉTODOS}

Nossa casuistica consta de 5 pacientes com ausências mioclônicas que, por ocasião de sua internação, apresentavam um ou vários episódios por dia.

Planejamento clinico-terapêtico - A seleção dos casos foi baseada em dados clínicos e na capacidade do doente em relatar com precisão o número de crises. Para verificar a exatidão das informações prestadas pelos doentes foram marcadas entrevistas preliminares. Nestas, pedia-se a cada paciente assinalar as crises eventualmente sofridas durante a consulta. As manifestações foram espontâneamente ou propositadamente precipitadas por hiperpnéia, estimulação luminosa intermitente ou emoções. Considerou-se resultado satisfatório a enumeração correta de 10 ou mais crises consecutivas ao longo de uma ou várias entrevistas. Uma vez considerado apto, o paciente foi cuidadosamente informado sôbre o plano de investigação clínico-terapêutica, ao qual seria submetido em regime de internação hospitalar durante três meses.

Após a internaçāo foi solicitado a cada paciente o registro, por escrito, das crises e de seus horários, bem como o aparecimento de qualquer nova manifestação. Foram anotadas as ocorrências de fatôres emocionais e de processos febris, o início e o final dos períodos menstruais. Durante o período de observaçoes preliminares, foi reavaliada a exatidão dos dados fornecidos pelos pacientes e foram realizados exames eletrencefalográficos durante a vigilia e o sono noturno fisiológico.

As medicações anticonvulsivantes usadas pelos pacientes foram gradativamente suspensas, exceção feita ao fenobarbital, mantido em uma dose única, à noite, durante todo o periodo experimental $(50 \mathrm{mg}$ para o caso 4 e $100 \mathrm{mg}$ para os casos 1, 2, 3 e 5). Foram empregadas, neste periodo, doses diárias progressivas (10, 20 e $30 \mathrm{mg}$ ) de diazepam e de nitrazepam para verificar a tolerância individual. Após os seis dias de administração, a medicação foi suspensa, no minimo, durante uma semana. Escolheu-se assim, a dosagem individual que não provocava sonolência indesejável.

Foram internados na mesma enfermaria, em duas oportunidades, dois pacientes com o mesmo quadro (casos 1 e 2 e casos 3 e 4), criando-se a possibilidade de contrôle mútuo das crises. Dessa forma foi possivel o registro fiel das crises do caso 4 por parte de sua mãe (caso 3 ). Outros pacientes, internados na mesma enfermaria foram instruídos no sentido de também assinalarem as crises que observavam.

As idades dos pacientes, por ocasião de sua internação, variavam entre 9 e 29 anos.

Os doentes receberam: diazepam, nitrazepam, trimetadiona $(3,5,5$ trimetiloxazolidina 2-4-dione) e um placebo (amido). As medicações foram colocadas em cápsulas idênticas com a finalidade de evitar seu reconhecimento por parte dos doentes e do Servico de Enfermagem. Os produtos foram administrados com intervalos de 6 horas, em doses de 10 ou $20 \mathrm{mg}$ de diazepam e de nitrazepam, e de $1,2 \mathrm{~g}$ de trimetadiona por dia.

Cada uma das medicaçōes foi administrada durante 4 dias consecutivos. $\mathrm{Cm}$ tratamento completo, utilizando as 4 medicações propostas tinha a duração de 16 dias; com o fim de se eliminar os possíveis efeitos residuais de uma droga sôbre outra, introduzida ulteriormente, os tratamentos foram repetidos três vêzes. Em cada um dos 4 tratamentos completos, cada droga era precedida e seguida por drogas diferentes. Precedendo e sucedendo as 4 séries de tratamento uma das drogas testadas, o diazepam foi administrado durante periodos de 4 dias. Na análise dos resultados êsses dois tratamentos não foram considerados. No esquema do planejamento, foi atribuída uma sigla a cada droga utilizada durante 4 dias consecutivos; a seqüência dos tratamentos foi: A/ABCD/ACBD/CCAD/DBBA/A. Nos diversos pacientes, as siglas B, C e D não corresponderam, necessàriamente, às mesmas drogas. 
Diàriamente as cápsulas foram pessoalmente entregues ao posto de Enfermagem, em envelopes nos quais constavam o nome do paciente e a data de administração da droga.

Técnica estatística - Na análise dos resultados, a comparação das médias individuais dos diversos tratamentos foi baseada no teste de Tukey, escolhendo-se o nível de significância de $5 \%^{7}$.

Características gerais dos doentes - Nossos pacientes não faziam referências a manifestações objetivas precedendo o distúrbio de consciência; em nenhum dêles ocorreu dissociação entre as mioclonias e as ausências (mioclonias sem ausência ou ausência sem mioclonias). A duração das manifestações críticas, observadas por ocasião das entrevistas preliminares e no decorrer da realização dos exames EEG, variava de 5 a 75 segundos. Durante as crises os pacientes não tinham capacidade de prosseguir atos iniciados antes da perda de consciência. Quando estas passavam, não se lembravam de acontecimentos particulares ou de perguntas feitas durante as mesmas. Ocasionalmente, contudo, relatavam ter percebido "repuxos" correspondendo às mioclonias. Referiam, uma vez cessado o episódio, ter tido a crise, embora, via de regra, não soubessem indicar com precisão as sensações subjetivas que possibilitavam o reconhecimento da manifestação paroxística. Freqüentemente um pequeno periodo de confusão era observado no período pós-crise; cefaléia e sonolência nunca foram assinaladas após as ausências mioclônicas.

A intensidade das mioclonias foi bastante variável; contudo, em cada um dos pacientes, os fenômenos motores que acompanhavam as ausências foram pràticamente iguais, em crises sucessivas. Foram observadas mioclonias limitadas à musculatura orbicular (caso 1); à musculatura facial e ocasionalmente dos membros superiores (caso 2); à musculatura facial e dos 4 membros, particularmente dos superiores ( $\operatorname{casos} 3,4$ e 5 ).

A freqüência aproximada das crises no decorrer das semanas que precederam o ensaio terapêutico e as idades em que surgiram estão indicadas no quadro 1 .

\begin{tabular}{ccc}
\hline Caso & Frequência das crises & Idade \\
\hline 1 & 2 a 6 & 14 anos \\
2 & 10 a 70 & 10 anos \\
3 & 1 a 30 & 15 anos \\
4 & mais de 25 crises por dia & 6 anos \\
5 & mais de 15 crises por dia
\end{tabular}

Quadro 1 - Freqüência diária aproximada das ausências mioclônicas antes do ensaio terapêtico. Idade em que surgiram.

Além das ausências mioclônicas foram referidas outras manifestaçōes epiléticas em três pacientes (casos 1, 4 e 5) e suas características estáo indicadas no quadro 2 .

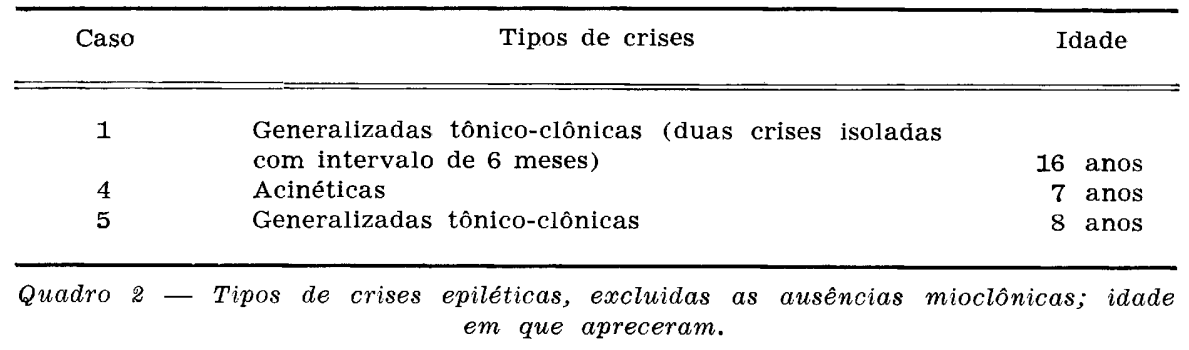


Em uma paciente (caso 1) havia antecedente de traumatismo de parto, traduzido por circular de cordão, cianose neonatal e demora de dois minutos para chorar. $O$ desenvolvimento psicomotor foi normal. Nos demais casos, a história, o exame neurológico e os exames subsidiários não permitiram, de momento, suspeitar uma possível etiologia. Afastou-se a possibilidade de moléstia hereditária de tipo degenerativo nos casos 3 e 4 (mãe e filho), pois, apesar da extrema semelhança do quadro epilético, não havia alteração do exame mental, do exame neurológico, nem manifestações epiléticas de tipos comumente encontrados em encefalopatias epiléticas degenerativas. Antecedentes pessoais e/ou familiares de teniase foram assinalados nos casos 1 e 2 .

Antes do inicio do ensaio terapêutico foram tentadas diversas medicações em todos os pacientes. No quadro 3 estão indicadas as medicações usadas e a duração dos tratamentos.

\begin{tabular}{clc}
\hline Caso & \multicolumn{1}{c}{ Medicação } & $\begin{array}{c}\text { Duracão do tra- } \\
\text { tamento em meses }\end{array}$ \\
\hline \hline 1 & Fenobarbital + primidona & 22 \\
2 & Fenobarbital + primidona + trinuride & 15 \\
& Fenobarbital + primidona & 26 \\
3 & Fenobarbital + difenil-hidantoina & 14 \\
& Fenobarbital + carbamoil-dibenzoazepina & 7 \\
& Fenobarbital + trimetadiona & $4^{*}$ \\
& Fenobarbital + primidona & $6^{*}$ \\
& Fenobarbital + trimetadiona & $4^{*}$ \\
& Fenobarbital + primidona & $6^{*}$ \\
& Fenobarbital + difenil-hidantoina & 32 \\
& Fenobarbital + primidona & 7 \\
\hline
\end{tabular}

Quadro 3 - Medicações anticonvulsivantes usuais e duração dos respectivos tratamentos antes do incio do ensaio terapêtico planejado nos 5 pacientes com ausências mioclônicas. * Tratamento irregular.

Nos 5 pacientes nenhuma das medicações prescritas exerceu influência significativa sôbre a intensidade e a frequiência das ausências mioclônicas. Houve contrôle das crises tônico-clônicas generalizadas com o uso de fenobarbital e primidona (caso 1); diminuição progressiva do mesmo tipo de crise foi observada com o uso de fenobarbital e difenil-hidantoina e, ulteriormente, de fenobarbital e primidona (caso 5).

o quadro de crises acinéticas não sofreu modificações com o emprêgo da associação fenobarbital + trimetadiona e, ulteriormente, de fenobarbital + primidona (caso 4).

Estudos eletrencefalográficos - Os principais achados eletrencefalográficos dos 5 pacientes com ausências mioclônicas estão indicados no quađro 4 . 
1 Atividade de fundo regular. Boa diferenciação espacial dos rítmos cerebrais. Complexos ponta-ondas de 3 c/seg difusos e síncronos, predominando nas projeções anteriores.

2 Atividade de fundo regular. Boa diferenciação espacial dos rítmos cerebrais. Complexos ponta-ondas e poliponta-ondas rápidas de $4 \mathrm{c} / \mathrm{seg}$ difusos e bilaterais.

3 Atividade de fundo discretamente irregular, polirritmica, sem diferenciação espacial evidente dos rítmos cerebais. Complexos ponta e poliponta-ondas de $3 \mathrm{c} / \mathrm{seg}$. Surtos de ondas monomorfas de $3 \mathrm{c} / \mathrm{seg}$ de projeção difusa.

4 Atividade de fundo irregular predominando ritmos de $7 \mathrm{c} / \mathrm{seg}$. Surtos de ondas de $3 \mathrm{c} / \mathrm{seg}$ de projeção posterior. Complexos poliponta-ondas difusos.

5 Atividade de fundo regular, com boa diferenciação espacial dos rítmo cerebrais. Complexos poliponta-ondas lentas de 2,5 a $3 \mathrm{c} / \mathrm{seg}$ de projeção difusa.
Complexos ponta-ondas mais freqüentes.

Complexos ponta ou poliponta-ondas mais freqüentes.

Complexos ponta ou poliponta-ondas mais freqüentes.

Complexos poliponta-ondas mais frequientes. Surtos difusos de ondas de $3 \mathrm{c} / \mathrm{seg}$.

Complexos poliponta-ondas lentas mais freqüentes.
Não há reação paroxística.

Reação paroxistica pouco intensa (ondas lentas posteriores).

Não há reação paroxística.

Intensa reação paroxística nas freqüências de 8 a 16 estimulos/seg.

Não há reação paroxística.
Acentuação das descargas durante os estádios I e II. Degra dação dos complexos ponta-ondas durante o estádio III. Desa. parecimento das descargas no estádio IV.

Surtos de pontas rítmicas e com menos freqüencia complexos po liponta - ondas durante os estádios I e II. Raros com complexos ponta ou poliponta - ondas durante o estádio IV.

Acentuação das descargas durante os estádios I e II. Desapare cimento no estádio IV.

Acentuacão e degradacão progressiva das descargas durante o sono lento. Desaparecimento das descargas durante o sono paradoxal.

Acentuação e degradação das descargas durante os estádios $\mathrm{I}$ e II. Desaparecimento das descargas durante o sono paradoxal.

Quadro 4 - Principais achados eletrencefalográficos nos 5 pacientes com ausências mioclónicas antes do inicio do ensaio terapêtico. 


\section{RESULTA DOS}

Em cada caso, o número de crises ocorridas durante os quatro tratamentos está indicado no quadro 5 .

\begin{tabular}{|c|c|c|c|c|c|}
\hline Caso & Nitrazepam & Diazepam & Trimetadiona & Placebo & $\begin{array}{l}\text { Total } \\
\text { geral }\end{array}$ \\
\hline 1 & 0 & 5 & 11 & 24 & 40 \\
\hline 2 & 54 & 141 & 243 & 417 & 855 \\
\hline 3 & 0 & 0 & 27 & 256 & 283 \\
\hline 4 & 2 & 6 & 215 & 1150 & 1373 \\
\hline 5 & 262 & 268 & 337 & 529 & 1396 \\
\hline
\end{tabular}

A simples inspeção dêste quadro permite, sem necessidade de análise estatistica, verificar que em dois casos ( 3 e 4) é possível separar os efeitos das diversas medicações. O conjunto dos tratamentos pelo nitrazepam e pelo diazepam proporcionou melhores resultados quanto ao contrôle das ausências mioclônicas. A terapêutica com trimetadiona ofereceu resultados menos satisfatórios, porém, nitidamente superiores aos obtidos com o placebo.

Os dados referentes aos casos 1, 2 e 5 foram analisados estatisticamente. A comparação entre as médias individuais dos diversos tratamentos foi feita pelo teste de Tukey (quadro 6).

\begin{tabular}{lccc}
\hline & Casos & & \\
Tratamentos & 1 & 2 & 5 \\
\hline \hline Nitrazepam & 0,0 & 3,4 & 16,4 \\
Diazepam & 0,3 & $\frac{8,9}{16,8}$ \\
Trimetadiona & 0,7 & $\frac{15,2}{21,1}$ \\
Placebo & 1,5 & $\frac{26,1}{33,1}$ \\
\hline \hline Quadrado médio do êrro & 0,61 & $\mathbf{2 8 , 8}$ & $\mathbf{9 0 , 0}$ \\
tsd & 1,12 & $\mathbf{7 , 7}$ & $\mathbf{1 3 , 5 5}$ \\
Valor de z & 1,87 & 2,22 & 2,031 \\
Relação de variância (F) & - & & $15,1+$ \\
\hline
\end{tabular}

Quadro 6 - Média do número de crises diárias durante a vigência do ensaio terapêutico com os vários medicamentos. As médias de cada coluna, separadas por traços horizontais, são significativamente diferentes das demais e foram separadas pelo teste de Tukey. $t s d$ e $z=$ parâmetros do teste. Valor de F para 2 e 48 graus de liberdade, ao nível crítico de $5 \%=3,19$. Valor crítico da distribuição normal ao nivel de $5 \%=1,96$. 
No caso 1 não se pode separar a média das crises ocorridas durante o tratamento com placebo da média das crises ocorridas durante os demais tratamentos. No caso 2 existe uma diferença significativa entre os resultados obtidos com o placebo e os obtidos com os demais tratamentos; prosseguindo a análise, foi possível separar o efeito da trimetadiona dos efeitos do diazepam e do nitrazepam. No caso 5, a análise estatística permitiu separar o efeito do placebo.

Prosseguindo-se a análise para os três tratamentos restantes, o valor de F obtido, para a relação de variância com 2 e 48 graus de liberdade, indica diferença significativa separando, provàvelmente, o efeito do tratamento com trimetadiona dos efeitos dos tratamentos com nitrazepam e diazepam.

Em três pacientes (casos 2, 3 e 5) ocorreram crises convulsivas tônico-clônicas generalizadas durante o seguimento terapêutico. A incidência de tais manifestações em relação aos diversos tratamentos está indicada no quadro 7 .

\begin{tabular}{llll}
$\begin{array}{c}\text { Casos } \\
\text { Tratamentos }\end{array}$ & 2 & 3 & 5 \\
\hline Nitrazepam & 2 & 0 & 1 \\
Diazepam & 2 & 0 & 1 \\
Trimetadiona & 1 & 0 & 0 \\
Placebo & 0 & 1 & 1 \\
\hline
\end{tabular}

Quadro 7 - Número de crises convulsivas tônico-clônicas generalizadas ocorridas em cada um dos tratamentos em três pacientes com ausências mioclônicas.

\section{COM E N T ÁRTOS}

Nossos resultados demonstram o efeito do diazepam e do nitrazepam sôbre as ausências mioclônicas.

Assim, em dois casos, não houve necessidade de análise estatística para demonstrar a superioridade dos derivados benzodiazepínicos sôbre os demais produtos utilizados, enquanto que, em mais dois, a aplicação do teste de Tukey leva à mesma conclusão.

Em apenas uma paciente (caso 1), a análise estatística não demonstrou diferenças significativas, provàvelmente devido ao pequeno número de crises que a mesma apresentava. No entanto, as flutuaçōes do número de manifestações paroxísticas no decorrer do ensaio terapêutico sugerem comportamento semelhante ao dos outros pacientes.

Apesar da análise estatística não demonstrar diferenças significativas entre as ações do diazepam e do nitrazepam, os dados obtidos durante o ensaio terapêutico sugerem ser o nitrazepam o mais eficaz. Em cada um dos 5 pacientes, o número total de crises, enquanto tratados com nitrazepam, nunca foi superior ao número de crises observadas durante a terapêutica com diazepam. Embora as diferenças individuais não sejam significativas, a repetição constante da tendência de mesmo sentido deve ser considerada a favor de uma diferença real de ação. 
O principal inconveniente dos derivados benzodiazepínicos reside na sua propriedade de favorecer o aparecimento de crises convulsivas tônico-clônicas generalizadas, fato evidente em três dos nossos pacientes. Convém salientar que as convulsões tônico-clônicas generalizadas surgiram, pela primeira vez, durante o ensaio terapêutico ( 2 e 3 ), apesar da cobertura barbitúrica. Embora tenhamos poucos elementos para chegar a uma conclusão definitiva, os dados obtidos, nos 5 pacientes submetidos ao presente estudo e em outros doentes com ausências mioclônicas, mas que por diversos motivos não participaram desta pesquisa, sugerem que não há diferença entre o nitrazepam e o diazepam quanto à propriedade de precipitar crises convulsivas generalizadas. A facilitação das crises generalizadas tônico-clônicas pelo diazepam está de acôrdo com a relativa ineficácia do produto contra as convulsões por eletrochoque ${ }^{10}$.

Essa mesma tendência também é observada em relação ao trimetadione, embora a análise de nossos protocolos sugira que os benzodiazepínicos tenham ação convulsivamente mais acentuada.

Durante o ensaio terapêutico foi possível constatar diminuição da intensidade das mioclonias que acompanhavam as ausências, quando os pacientes eram tratados com nitrazepam ou diazepam. Êsse efeito persistiu depois da alta hospitalar, quando o nitrazepam foi mantido. Em dois pacientes (casos 1 e 2) as mioclonias desapareceram, permanecendo as ausências. Achado semelhante não foi observado com o uso da trimetadiona.

Além do efeito satisfatório do nitrazepam e do diazepam sôbre as ausências mioclônicas, é possível verificar a ação, embora limitada, da trimetadiona sôbre êsse tipo de crise; revelou-se, em todos os casos, superior ao placebo, porém sempre inferior ao nitrazepam e ao diazepam. Essas diferenças foram evidentes, sem necessidade da análise estatística, em dois casos e, após a aplicação do teste de Tukey, em mais dois. No caso 1, embora a análise estatística não revele diferenças significativas, os dados numéricos sugerem comportamento semelhante.

Deve-se notar que dois pacientes (casos 3 e 4) tinham sido medicados antes do ensaio terapêutico com trimetadiona, sem melhora clínica. A dosagem foi inferior àquela usada durante o teste terapêtico $(0,6 \mathrm{~g}$ por dia $)$, o que pode ter contribuido para a não observação, por parte dos pacientes, de melhora evidente. Não se pode afastar a possibilidade, contudo, de ter havido diminuição real do número de crises, mas de tal ordem que passou desapercebida. Estudos semelhantes ao que realizamos permitirão melhor avaliação do efeilto de drogas sôbre a incidência de manifestações paroxísticas.

Os dados obiidos correspondem a um limitado período de observação. Devem ser complementados por estudos longitudinais que permitirão verificar se, a longo prazo, os efeitos do diazepam e do nitrazepam permanecem, ou não. Após mais ảe um ano de observação dos efeitos da associação nitrazepam-fenobarbital (complementada em dois pacientes com outras drogas, de ação sôbre crises convulsivas generalizadas), pudemos observar o contrôle das crises em dois (casos 3 e 4) por período superior a 6 meses e sua re- 
dução considerável em mais dois (casos 1 e 2). Êstes últimos pacientes ainda apresentam até três crises por dia, passando mais de uma semana sem manifestações que, quando ocorrem, são freqüentemente relacionadas com fatôres emocionais. Em apenas um doente os resultados a longo prazo são decepcionantes, tanto pela ocorrência diária de ausências mioclônicas em número freqüentemente superior a 40 , quanto pelo aparecimento de crises generalizadas não controladas, mesmo com doses crescentes de primidona e de difenil-hidantoina. Os casos que apresentam associação de ausências com crises convulsivas generalizadas constituem ainda difícil problema terapêutico.

\section{RESUMO E CONCLUSOEES}

Foi comparada a potência anticonvulsivamente do nitrazepam, do diazepam e da trimetadiona em 5 pacientes com ausências mioclônicas. Os doentes receberam, além das medicações anticonculsivantes, um placebo.

O teste terapêutico foi constituído por tratamentos sucessivos de 4 dias, planejados com o fim de se eliminar os possíveis efeitos residuais de uma droga sôbre a outra, administrada ulteriormente. $\mathrm{Na}$ análise dos resultados, a comparação das médias individuais dos diversos tratamentos foi baseada no teste de Tukey.

Em dois casos não houve necessidade de análise estatística para demonstrar a superioridade do nitrazepam e do diazepam sôbre os demais produtos utilizados; em outros dois, a aplicação do teste de Tukey levou à mesma conclusão. No quinto caso as flutuações do número de ausências mioclônicas durante o ensaio terapêutico sugerem comportamento semelhante, embora a análise estatística individual não revele diferenças significativas. Há indícios de que o nitrazepam tem ação superior ao diazepam sôbre êsse tipo de crise convulsiva.

A trimetadiona revelou-se superior ao placebo, porém inferior ao nitrazepam e ao diazepam.

O principal inconveniente do nitrazepam e do diazepam é o de precipitar crises generalizadas tônico-clônicas.

S U M M A R Y

Myoclonic absences: a comparative study of the anticonvulsant potency of nitrazepan and diazepan

The anticonvulsant potency of nitrazepan, diazepan and trimetadiones in five patients with myoclonic absences was compared. A placebo was also used. Therapeutic tests were performed by 4-days successive treatments designed to avoid the residual effects of one drug upon the other administered afterwards. In two cases statistical analysis was not necessary for demonstration of the superiority of nitrazepan and diazepan in regard to trimetadiones; in two others the application of Tukey's test allowed this 
same conclusion. In the last case the numeric variations of the seizures during the therapeutic essay suggest identical conclusion allthough the statistical analysis was not significant. There are indications that nitrazepan is more effective than diazepan against this type of epileptic seizures.

\section{REFERENCIAS}

1. ARMBRUST-FIGUEIREDO, J. - Codificação de terapêutica das síndromes epilépticas para avaliação dos efeitos imediatos e tardios de determinadas drogas. III Congresso Brasileiro de Neurologia, Recife, julho de 1968.

2. BIOảảEY, D. - Epilepsie myokinétique de la première enfance avec pointe-onde lent: syndrome de Lennox. Acta neurol. psychiat. Belg. 67:599, 1967.

3. GASTAUT, H.; ROGER, J.; LOB, H. \& TASSINARI, C. A. - Les nouveaux médicaments anti-épileptiques. Encéphale 54:407, 1965.

4. GASTAUT, H.; ROGER, J.; LOB, H. \& TASSINARI, C. A. - L'action du diazépam (Valium) dans le traitement des formes non convulsives de l'épilepsie généralisée (absences et états d'absences de types divers). Sem. Hôp. Paris 41:1541, 1965.

5. GIBBS, F. A. \& ANDERSON, E. M. - Treatment of hypsarhythmia and infantile spasms with a Librium analogue. Neurology (Minneapolis) 15:1173, 1965.

6. LISKE, E. \& FORSTER, F. M. - Clinical study of a new benzodiazepine as an anticonvulsivant agent. J. New Drugs (New York) 3:241, 1963.

7. LISON, L. - Statistique Appliquée à la Biologie Expérimentale: la Planification de l'Expérience et l'Analyse des Résultats. Gauthier-Villars, Paris, 1958.

8. LISON, M. P. \& MEGA, D. - Espasmos em flexão: estudo clínico e eletrencefalográfico de pacientes tratados com Ro 4:5360. Arq. Neuro-Psiquiat. (São Paulo) 25:87, 1967.

0. MARKHAM, C. H. - The treatment of myoclonic seizures of infancy and childhood with LA-1. Pediatrics 34:511, 1964.

10. MEHMET EKINSKI, HSU, J. J.; BRUK, M. \& BRAUN, R. A. - Diazepam as an anticonvulsivant agent in eletroshock therapy. Am. J. Psychiat. 120:903, 1964.

11. MILliCHAP, J. G. \& ORTIZ, W. R. - Nitrazepam in myoclonic epilepsies. Am. J. Dis. Child. 112:242, 1966.

12. PETERSON, W. G. - Clinical study of Mogadon. A new anticonvulsant. Neurology (Minneapolis) 17:878, 1967.

13. TROLLE, E. - Diazepam (Valium) in the treatment of epilepsy. A report of fifty cases. Acta neurol. Scandinav. 41:535, 1965.

14. VoLZKE, E.; DOOSE, H. \& STEPHAN, E. - The treatment of infantile spasms and hypsarhythmia with Mogadon. Epilepsia (Amsterdam) 8:64, 1967.

15. WEINMAN, H. M. - Le traitement des crises infantiles par le Mogadon. Rev. Neurol. (Paris) 117:152, 1967.

16. WHITE, P. T.; PLOTT, D. \& NORTON, J. - Relative anticonvulsant potency of primidone. Arch. Neurol. 14:31, 1966.

Departamento de Neurologia - Faculdade de Medicina de Ribeirão Prêto - Ribeirão Prêto, SP. - Brasil. 